\title{
Mentoría en curso de emprendimiento en línea. Sistematización de una experiencia en educación superior
}

\author{
May Portuguez-Castro ${ }^{1}$ y Marcela G. Gómez-Zermeño ${ }^{\star}$ \\ Escuela de Humanidades y Educación, Tecnologico de Monterrey, Monterrey, México. \\ (correo-e: mayportuguezc@gmail.com; marcela.gomez@tec.mx) \\ ${ }^{*}$ Autor a quien debe ser dirigida la correspondencia
}

Recibido Abr. 9, 2020; Aceptado Jun. 5, 2020; Versión final Jul. 22, 2020, Publicado Dic. 2020

\begin{abstract}
Resumen
El objetivo del presente estudio consistió en analizar los elementos que intervinieron en la mentoría de un curso en línea de emprendimiento con 20 estudiantes universitarios de carreras profesionales de una universidad del norte de México. En este estudio se siguió una metodología de sistematización de la experiencia educativa para profundizar en los pasos seguidos por los participantes en el proceso de mentoría y en la forma de realizar propuestas de solución. El análisis de los resultados mostró que, de cinco equipos participantes, los tres equipos que trabajaron en sesiones de mentoría realizaron una solución más estructurada que los otros dos grupos que tuvieron poca interacción con el mentor. Se identificaron obstáculos que impiden alcanzar los objetivos educativos, como son la falta de planificación del mentor y de tiempo de los estudiantes. Se concluye que las actividades descritas y los pasos sugeridos en esta investigación pueden replicarse tomando en cuenta acciones para mitigar los problemas presentados.
\end{abstract}

Palabras clave: mentoría; educación emprendedora; sistematización de la experiencia; aprendizaje en línea; innovación educativa; educación superior

\section{Mentoring in an online entrepreneurship course. Systematization of an experience in higher education}

\begin{abstract}
The main objective of the present study was to analyse the elements involved in mentoring an online entrepreneurship course that had 20 undergraduate students from a university in northern Mexico. The study followed a methodology of systematization of the educational experience to delve into the steps followed by the participants in the mentoring and solution making processes. The results showed that, of the five participating teams, three teams that worked in mentoring sessions made a more structured solution than the other two groups that had little interaction with their mentors. The systematization of this experience allowed identifying obstacles that prevent reaching educational objectives such as the lack of mentor planning and student time. It is concluded that the activities and the steps suggested in this research study can be replicated as longs as specific actions are taken to mitigate the issues described.
\end{abstract}

Keywords: mentoring; entrepreneurship education; experience systematization; online learning; educational innovation; higher education 


\section{INTRODUCCIÓN}

En los últimos años el tema del emprendimiento ha despertado interés, debido a que es considerado como una herramienta que contribuye al crecimiento económico, a dinamizar el proceso innovador y a generar nuevos empleos (Sánchez et al., 2017; Nabi et al. 2018). En un mundo globalizado los mercados deben adaptarse a nuevos esquemas, por lo que se requiere la formación de capacidades emprendedoras en las personas que permitan disminuir el impacto del cambio y lograr una sociedad con mejores oportunidades para todos (Scott-Kemmis, 2017). Esta formación debe contar con ciertas características que desarrollen a ciudadanos más activos, autónomos y orientados a la acción y estar basadas en modelos que sustenten esta formación (Hägg y Kurczewska, 2016). Sin embargo, los programas de educación para el emprendimiento requieren aún de mayor investigación, debido a que es necesario determinar lo que se debe enseñar y cómo hacerlo, pues no siempre se tienen claros los contenidos a estudiar ni los modelos pedagógicos adecuados para su enseñanza (Padilla-Meléndez et al., 2014; Nabi et al. 2019).

La educación desempeña un papel primordial para desarrollar las habilidades de emprendimiento, por lo que la formación en estas habilidades debe incluirse en todas las etapas del proceso educativo (OCDE, 2018). A nivel global las iniciativas para mejorar las competencias de emprendimiento se han incrementado y cada vez más, diferentes actores como las universidades, están incluyendo programas de formación emprendedora para desarrollar el talento innovador (Portuguez et al., 2019). Estudios anteriores han analizado de forma empírica los resultados de estas experiencias formativas. Por ejemplo, la investigación realizada por PadillaMeléndez et al. (2014) en una universidad de España, que buscaba conocer cómo desarrollar a través del aprendizaje experiencial, la intención emprendedora en los estudiantes. Como parte de su formación los alumnos realizaban juegos de roles, con el fin de simular situaciones reales de emprendimiento y solucionar problemas. Las conclusiones indicaron que aún se requiere analizar el diseño pedagógico de los cursos de emprendimiento para lograr mejorar la intención emprendedora, que lleva a disminuir la aversión al riesgo.

Un estudio realizado en una universidad superior en Colombia muestra los resultados de una encuesta aplicada a 540 estudiantes que han recibido formación en emprendimiento durante su carrera. Su objetivo fue analizar la relación entre la interdisciplinariedad y las evaluaciones en innovación e impacto en la sociedad de las ideas de negocio desarrolladas durante los cursos (Contreras-Velásquez et al. 2017). Los autores concluyeron que en los grupos en los que hay estudiantes de diferentes disciplinas, se obtuvo una evaluación mayor en innovación y un mayor impacto en la sociedad que en los de la misma disciplina. Por lo que consideran necesario que las universidades diseñen programas de formación en emprendimiento de manera transversal y que se aplique a todos los niveles académicos para favorecer el aprendizaje interdisciplinar (Contreras-Velásquez et al. 2017).

Otros estudios realizados en universidades de Chile analizaron los programas de emprendimiento existentes en esas instituciones. Los autores mencionan que para fomentar la cultura emprendedora es necesario que los centros de estudio diseñen programas de emprendimiento que incluyan el uso de recursos computacionales y el desarrollo de las habilidades emprendedoras. Se encontró que las universidades sí se preocupan por tener programas formativos en emprendimiento, sin embargo, faltan estudios que evalúen sus resultados y que vayan más allá de la obtención del grado del alumno (Herrera y Villalobos, 2018). También se considera importante establecer estrategias que fortalezcan los ecosistemas de emprendimiento, que lleven al alumno a desarrollar iniciativas extracurriculares de manera más activa (Caban et al. 2018).

Una de las metodologías que se utiliza para formar en emprendimiento es la mentoría (Lackéus, 2015), que es una manera de acercar al estudiante con el desarrollo de sus habilidades a través de la participación en actividades extracurriculares acompañado por un mentor, que les permiten conocer experiencias reales de emprendimiento y así aumentar su motivación para crear nuevas empresas (Bell y Bell, 2016). El rol del mentor tiene relevancia para el alumno, ya que favorece la motivación del estudiante y brinda apoyo a la experiencia educativa gracias a los aportes basados en sus propias experiencias. Según los autores consultados, la mentoría puede ser realizada de tres maneras: a través de emprendedores exitosos a emprendedores aprendices, haciendo a los aprendices mentores de otros estudiantes, o asesorándose en otras áreas del proceso de aprendizaje como negocios o banca (Lefebvre y Redien-Collot, 2013; Gimmon, 2014). Aunque generalmente los mentores son personas con gran experiencia en el desarrollo de emprendimientos (Rigg y O'Dwyer, 2012). Además, se recomienda que sea impartida no solo en las facultades de negocio, sino que debe darse a través de las diferentes carreras (Bell y Bell, 2016).

En estudios recientes relacionados con la aplicación de la mentoría en educación para el emprendimiento, se considera importante para el éxito de su implementación, la intención del mentor de compartir sus experiencias, así como la capacidad e intención del aprendiz en aprender de éstas, donde exista interacción entre los involucrados y se establezca una relación a través de una comunicación efectiva (Sharafizad, 2017; Ting et al., 2017). En este sentido, la labor del mentor consiste en orientar a los estudiantes a través de sus 
experiencias, para que los aprendices tengan más capacidad para resolver problemas, incluso cuando termine el programa de formación (Sendouwa et al., 2019). Para Babatunde y El-Gohari (2019) la mentoría trae beneficios para los aprendices y los mentores: para los estudiantes, los conocimientos compartidos les permiten lograr con éxito los objetivos planteados y para los mentores, los beneficios consisten en mejorar la comunicación con generaciones de empresarios más jóvenes, reforzar los conocimientos y desarrollar habilidades de escucha y retroalimentación.

Nabi et al. (2019) proponen que la mentoría debe ser un proceso que tome en cuenta las características particulares del estudiante, más que desarrollar un enfoque genérico, para apoyar a los alumnos en su transición de estudiante a emprendedor. También que la mentoría sea un apoyo socioemocional para reducir la ansiedad que produce el iniciar un emprendimiento, donde el mentor no solo muestre cuál es la forma de hacer las cosas, sino que brinde la confianza para favorecer la autoeficacia hacia la formación del espíritu empresarial. Se encuentra que en la literatura existen pocos estudios empíricos que hayan explorado la mentoría en educación para el emprendimiento en ambientes universitarios (Kubberød et al., 2017).

Además, de acuerdo con una revisión sistemática de literatura realizada por Tinoco-Giraldo et al. (2020) sobre las actividades de mentoría en línea con estudiantes universitarios, se encuentra que existen diversos problemas para su implementación debido a la falta de definición de estrategias que permitan una mentoría efectiva, así como no existe información clara sobre instrumentos que evalúen los factores involucrados en este proceso de enseñanza-aprendizaje.

\section{OTROS ANTECEDENTES}

Con el desarrollo de las nuevas tecnologías cada vez más los procesos educativos hacen que los roles del docente y del alumno cambien y el caso de la educación para el emprendimiento no es la excepción, especialmente por los retos que trae la automatización y los requerimientos de la Cuarta Revolución Industrial. Así, deben surgir nuevas estrategias y políticas para la innovación educativa que permitan la adquisición de las competencias requeridas para una participación activa del estudiante. Estas competencias se caracterizan como: transversales, que no están directamente relacionadas a un campo en específico sino a varios campos y multidimensionales que incluyen diferentes habilidades, actitudes y conocimientos (Contreras-Velásquez et al. 2017). Por lo tanto, la aplicación de la innovación educativa en la educación para el emprendimiento presenta un cambio de paradigma para el logro de estas competencias, debido a que además de favorecer la creatividad y el trabajo multidisciplinario, también permite el dominio de las áreas científico-tecnológicas y el uso de las Tecnologías de Información y Comunicación (TIC) en ambientes más activos y colaborativos (Portuguez et al., 2019).

Como se ha encontrado en la literatura, existe aún la necesidad de desarrollar más estudios relacionados con la educación emprendedora, en los que se analicen temas asociados a la forma de enseñar el emprendimiento, que se favorezca el aprendizaje interdisciplinar y para el caso de la mentoría, definir estrategias especialmente para ambientes virtuales. Por lo tanto, este estudio busca analizar los elementos que intervinieron en la mentoría de un curso en línea de emprendimiento con estudiantes universitarios de carreras profesionales, a través de una sistematización de la experiencia educativa para poder ser replicada en otros cursos. En este sentido se sigue la metodología de sistematización de la experiencia educativa para analizar los pasos seguidos por los participantes en el proceso de mentoría y en la forma de realizar propuestas de solución. Esta propuesta puede ser utilizada en otros entornos y también modificarse según las posibilidades de la institución. El rol del mentor puede ser asumido por otros profesores o por los mismos estudiantes siguiendo las pautas establecidas en este estudio.

La metodología de la sistematización de la experiencia educativa busca comprender la experiencia, extraer sus enseñanzas y compartirlas, realizando una interpretación crítica del proceso vivido por los participantes (Jara, 2018). Esta experiencia corresponde a los resultados del proceso de mentoría realizado durante un curso en línea sobre emprendimiento con alumnos en una universidad del norte de México. Esta universidad cuenta con un ecosistema emprendedor que incluye incubadoras, aceleradora de empresas, parques tecnológicos, institutos de emprendimiento, oficinas de transferencia tecnológica y de apoyo a emprendedores, además se ofrecen programas de emprendimiento tanto curriculares como extracurriculares. Todas las actividades extracurriculares y los servicios del ecosistema emprendedor son opcionales y su duración depende del tipo de programa y el nivel de emprendimiento que se alcance. Además, los costos también dependen del programa y del tiempo que invierta el emprendedor en participar de estos programas.

A continuación, el presente artículo está estructurado de la siguiente manera: primero se presenta la metodología en la que se describe la manera en que se realizó el estudio, los participantes, los instrumentos utilizados y la forma en que se analizaron los datos. Después se presentan los resultados según la metodología del estudio, la discusión de los resultados contrastándolos con la literatura consultada y por último las conclusiones. 


\section{METODOLOGÍA}

El estudio se enmarca en la investigación cualitativa donde se busca la descripción e interpretación de la experiencia (Picado-Alfaro, 2018). Para analizar las experiencias en el proceso de mentoría de los alumnos se realizó una sistematización de la experiencia educativa. La sistematización se define como el análisis de las prácticas educativas a través de la observación y el registro de la experiencia, con la finalidad de comprender y profundizar en los aprendizajes de los participantes y sus interacciones (Barbosa-Chacón et al., 2015; Inciarte-González et al. 2017). Para realizar este proceso Jara (2018) declara que se debe reflexionar de manera individual y colectiva, alrededor de un objeto de la sistematización, para luego reconstruir la experiencia de manera cronológica según lo ocurrido, realizando una mirada crítica para generar nuevos conocimientos sobre lo vivido.

La metodología de sistematización de experiencias educativas sigue el paradigma cualitativo y se encuentra dentro del enfoque de investigación acción participativa, con un diseño que se aproxima a la etnografía y con un alcance transversal (Falkembach y Torres, 2015). Su objetivo es explorar los contextos educativos para obtener descripciones que expliquen la realidad subjetiva de las prácticas de una manera sociocrítica (Barbosa-Chacón et al., 2015). Si bien en sus inicios esta metodología fue objeto de debate, cada vez más autores la utilizan para describir y explicar los fenómenos educativos. Se considera como una estrategia adecuada para desarrollar en este estudio debido a que permite a través de una serie de pasos, documentar y reflexionar sobre las experiencias vividas en el proceso de enseñanza aprendizaje para llegar a una etapa de análisis y reflexión de la información (Jara, 2018). El proceso de sistematización de experiencias se realiza en etapas o fases. Para llevar a cabo la sistematización de la experiencia educativa de la mentoría se siguieron los pasos recomendados por Jara (2018) para elaborar una propuesta que permita ser desarrollada en este contexto, como se muestra en la Figura 1.

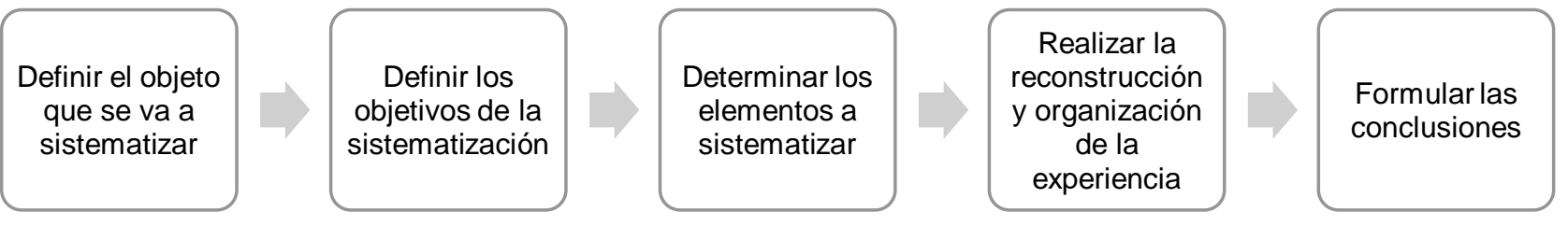

Fig. 1: Proceso de sistematización de la experiencia educativa. (Adaptada de Jara, 2018)

\section{Plan de la sistematización de la experiencia}

El primer paso de la sistematización es la definición del objeto que se va a sistematizar. En este estudio el objeto de la sistematización consistió en las experiencias educativas realizadas en el proceso de mentoría, durante un curso en línea realizado con estudiantes universitarios de nivel profesional de diferentes carreras. Este curso en línea busca que, por medio de pedagogías innovadoras y herramientas tecnológicas, se genere conocimiento hacia el diseño de estrategias que permitan identificar a los participantes sus habilidades de emprendimiento. En esta experiencia formativa el proceso de mentoría tiene la finalidad de fortalecer el desarrollo de ideas de solución a problemas sociales. Esta etapa del curso se llevó a cabo durante tres semanas en una plataforma virtual con 25 participantes, 20 alumnos de diferentes carreras de una universidad en el norte de México y 5 mentores que son emprendedores reales, relacionados con la formación de emprendedores y que se encuentran en diferentes estados de México.

El segundo paso consiste en definir los objetivos de la sistematización. Éstos se establecieron como:1) describir las formas en que los participantes se organizan para realizar las actividades; 2) identificar los medios que utilizaron los participantes para comunicarse; y 3) analizar los elementos de mentoría presentes en la experiencia. El siguiente paso consiste en determinar los elementos a sistematizar. En este caso se eligieron tres elementos que permitieran conocer el desarrollo del proceso de la mentoría y sus resultados: 1) las interacciones entre los participantes para cumplir con las actividades; 2) los elementos de la mentoría observados en la experiencia; y 3 ) los reportes del mentor y alumnos sobre los resultados de la experiencia educativa.

Para realizar la reconstrucción y organización de la experiencia se organizaron de forma cronológica los hechos que se presentaron, dando especial interés a los acontecimientos relevantes y diferentes ritmos que se dieron en la actividad. Luego se organizó la información según los elementos centrales a sistematizar realizando una descripción de lo presentado. Posteriormente se plantearon preguntas críticas para poder identificar las causas de lo sucedido, identificar la lógica de lo ocurrido, determinar semejanzas y contradicciones y establecer relaciones con las teorías. Estas preguntas son las siguientes: ¿cuáles fueron los ritmos de trabajo?; ¿qué medios utilizaron para reunirse?; ¿cuáles fueron los principales acuerdos?; ¿qué 
elementos de la mentoría se dan?; ¿cuáles fueron los resultados obtenidos?; y ¿cuáles fueron las posibles causas de esos resultados? Por último, se formularon las conclusiones para responder a los objetivos de la sistematización, señalando los aciertos y desaciertos de esta implementación.

\section{Participantes}

El total de participantes es de 25 personas. Para participar en este estudio se invitó a 20 estudiantes de diferentes carreras de nivel profesional a realizar un curso en línea donde realizarían actividades para fortalecer sus habilidades de emprendimiento. En este curso participaron cinco mentores que fueron invitados a participar de un grupo de exalumnos de una maestría en comercialización de ciencia y tecnología y que contaran con experiencia en el desarrollo de emprendimientos. La muestra fue intencional y la participación fue voluntaria.

El perfil de los estudiantes corresponde a un $60 \%$ mujeres y un $40 \%$ hombres. Se encuentran en un rango de edad entre los 19 a 31 años, con un promedio de 22.86 años, todos son solteros y viven en Monterrey, México. Únicamente el $20 \%$ trabaja actualmente y un $5 \%$ ha tenido o tiene su propio negocio. Dentro de la carrera que estudian, el $60 \%$ cursa carreras relacionadas con ingeniería (12), de los cuales siete son mujeres y cinco hombres; el 30\% cursa la carrera de administración de empresas (4 mujeres y 2 hombres), 5\% diseño industrial (un hombre) y $5 \%$ psicología clínica (1 mujer). Todos los alumnos se encuentran cursando entre el 5 to y 9 no semestre de la carrera, estando el $80 \%$ en los últimos semestres de la carrera ( $7 \mathrm{mo}$ a 9 no semestre) y el resto en 5 to y 6 to semestre. $45 \%$ de los estudiantes mencionaron no haber llevado ningún curso de emprendimiento, $25 \%$ únicamente uno, $20 \%$ dos cursos y $10 \%$, 3 o más. Un $90 \%$ manifestó tener mucho o suficiente interés en ser emprendedor. El 100\% han utilizado plataformas en línea para sus cursos.

En cuanto a los mentores participantes, tres son mujeres y 2 hombres. Se encuentran en un rango de edad entre los 28 a 68 años, con un promedio de edad de 48.4 años. Todos cuentan con posgrado y tienen un promedio de 18 años de experiencia en la realización de emprendimientos. El 100\% de los mentores han sido emprendedores. Han trabajado en el campo de la investigación, docencia y desarrollo de empresas de base tecnológica y cuentan con especialidades de gestión de ciencia y tecnología, innovación, productividad y comercialización de ciencia y tecnología.

\section{Instrumentos}

Los instrumentos para recopilar la información fueron: 1) un cuestionario para determinar el perfil de los participantes, donde se presentaron preguntas relacionadas a la edad, género, nivel de estudios, experiencia, carrera que estudian y semestre cursado; 2) una matriz de observación donde se registró información relacionada con las reuniones, participaciones en chats y foros, grabaciones de las reuniones y entrevistas y 3) reportes de la mentoría donde se registraron las opiniones de los participantes y la retroalimentación sobre la actividad.

El reporte de la mentoría consistió en cuatro preguntas abiertas para los mentores y para los alumnos. A los mentores se les preguntó: 1) ¿en qué consistió la mentoría?; 2) ¿cuáles fueron los aprendizajes de los alumnos?; 3) ¿cuál es su opinión sobre el trabajo de los alumnos? y 4) ¿cuáles fueron los obstáculos que se presentaron y qué recomendaciones darían? A los alumnos se les consultó: 1) ¿qué aprendieron del mentor que participó en el curso?; 2) ¿cuáles problemas enfrentaron y cómo los manejaron?; 3) ¿qué les gustó más del curso? y 4) ¿qué no les gustó del curso?

\section{Análisis de datos}

El análisis de datos cuantitativos como la cantidad de interacciones en foros y en WhatsApp se realizó por medio de análisis de frecuencias y fueron representados con gráficas para mostrar comparaciones de los resultados. Por otro lado, para los datos cualitativos, como las participaciones en los foros y en las interacciones con el mentor en los grupos de WhatsApp y respuestas a los reportes sobre la mentoría, se realizó un análisis de contenido para buscar relaciones en las respuestas de los estudiantes. Por medio de un análisis inductivo, se determinaron categorías de análisis y se identificaron pasajes dentro de los textos que se consideraron relevantes para evidenciar el trabajo realizado por los participantes en el curso utilizando el software MAXQDA 2020. Para asegurar el rigor científico se realizó la triangulación de las respuestas de los mentores y los estudiantes a las preguntas del reporte de la mentoría, con los resultados de la matriz de observación que se llevó a cabo durante la investigación.

\section{RESULTADOS}

A continuación, se presentan los resultados de la sistematización de la experiencia educativa de la mentoría en un curso en línea de emprendimiento, producto del análisis de los datos obtenidos en los instrumentos. Debido a que la sistematización de experiencias pone énfasis en el proceso vivido durante la experiencia 
concreta y busca describir y entender qué es lo que sucedió y por qué ocurrió, en este estudio se buscó poder describir cómo vivieron los participantes este proceso y extraer lecciones qué permitan mejorarlos en una futura experiencia (Jara, 2018; Picado-Alfaro, 2018).

\section{Reconstrucción y organización de la experiencia}

Para iniciar con la mentoría se enviaron mensajes a los mentores para que aceptaran la participación en el estudio. Se les envío un cuestionario en línea en el que se explicaba el objetivo de su participación y que indicaran su aceptación en el mismo. La mentoría consistió en participar en un curso en línea en el que se les pide a los estudiantes proponer una solución a un problema de la comunidad y hacer una presentación, donde el rol del mentor es el de orientarlos en su solución. También se invitó a participar a estudiantes de diferentes carreras de nivel profesional. De los 20 estudiantes que comenzaron el curso, 12 alumnos eligieron un tema de interés para trabajar en una propuesta de solución y presentaron una propuesta. El grupo se dividió en 5 equipos y a cada mentor le correspondió un grupo. El equipo 1 trabajó el tema de proponer una solución para la seguridad energética; el equipo 2 para facilitar la apertura de negocios; el equipo 3 para identificar las causas de la corrupción; el equipo 4 para reducir la pobreza y el equipo 5 para mejorar la calidad del aire. La caracterización de los equipos se encuentra en la Tabla 1.

Tabla 1: Composición de los equipos de trabajo

\begin{tabular}{|c|c|c|c|}
\hline $\begin{array}{l}\text { Temática } \\
\text { abordada }\end{array}$ & Equipo & Integrantes & Mentor(a) \\
\hline $\begin{array}{l}\text { Seguridad } \\
\text { energética }\end{array}$ & 1 & $\begin{array}{l}1 \text { alumno de ingeniería química- } 7 \text { mo semestre. } \\
1 \text { alumno de ingeniería en mecatrónica- } 9 \text { no } \\
\text { semestre. }\end{array}$ & $\begin{array}{l}\text { Con especialidad en desarrollo } \\
\text { tecnológico y administración en } \\
\text { innovación con } 40 \text { años de } \\
\text { experiencia. }\end{array}$ \\
\hline $\begin{array}{l}\text { Facilitar la } \\
\text { apertura de } \\
\text { negocios }\end{array}$ & 2 & $\begin{array}{l}1 \text { estudiante de ingeniería física- } 7 \mathrm{mo} \text { semestre. } \\
1 \text { estudiante en ingeniería química- } 7 \mathrm{mo} \\
\text { semestre. } \\
1 \text { estudiante de ingeniería en mecatrónica- } 7 \text { mo } \\
\text { semestre. }\end{array}$ & $\begin{array}{l}\text { Con especialidad en productividad } \\
\text { y calidad, comercialización de } \\
\text { tecnologías con } 8 \text { años de } \\
\text { experiencia. }\end{array}$ \\
\hline $\begin{array}{l}\text { Causas de la } \\
\text { corrupción }\end{array}$ & 3 & 2 estudiantes de ingeniería civil- 5to semestre & $\begin{array}{l}\text { Especialidad en gestión de ciencia } \\
\text { y tecnología con } 10 \text { años de } \\
\text { experiencia. }\end{array}$ \\
\hline $\begin{array}{l}\text { Reducción de } \\
\text { la pobreza }\end{array}$ & 4 & $\begin{array}{l}1 \text { estudiante de psicología clínica- } 7 \text { mo semestre } \\
1 \text { estudiante de biotecnología- } 7 \text { mo semestre }\end{array}$ & $\begin{array}{l}\text { Con especialidad en innovación y } \\
\text { emprendimiento con } 11 \text { años de } \\
\text { experiencia. }\end{array}$ \\
\hline $\begin{array}{l}\text { Mejorar la } \\
\text { calidad del aire }\end{array}$ & 5 & $\begin{array}{l}1 \text { estudiante de ingeniería industrial- } 7 \mathrm{mo} \\
\text { semestre } \\
1 \text { estudiante de ingeniería en innovación y } \\
\text { desarrollo- } 7 \mathrm{mo} \text { semestre } \\
1 \text { estudiante de ingeniería en biotecnología- } 7 \mathrm{mo} \\
\text { semestre }\end{array}$ & $\begin{array}{l}\text { Con especialidad en } \\
\text { comercialización en ciencia y } \\
\text { tecnología con } 1 \text { año de } \\
\text { experiencia. }\end{array}$ \\
\hline
\end{tabular}

\section{Guía del mentor}

Se pidió a los mentores recolectar evidencias de lo trabajado en el curso y se dieron indicaciones de cómo organizarse. Sin embargo, la forma de resolver el problema y los elementos de solución se dejaron a discreción de cada grupo para conocer las alternativas que pueden ser utilizadas en un ejercicio de esta naturaleza. Se elaboró una guía en la que se indicaron aspectos como: qué es un mentor, el tipo de mentoría que se desarrolla en el curso, los medios para comunicarse (videoconferencia, chat), los roles del mentor y las actividades a realizar. En este curso los alumnos están invitados a participar de un reto, en el que se espera que pueden elegir un problema de su entorno y proponer un producto o servicio que lo solucione a través de una idea innovadora que pueda generar un emprendimiento. Para colaborar con el logro de este objetivo se esperaba que los mentores pudieran orientar a los alumnos en su definición de la idea, escuchar sus opiniones y retroalimentarse con aportes relacionados a su experiencia.

Actividades a realizar en el curso

Las actividades que se solicitaron realizar a los mentores fueron: 1) participar en un foro de presentación de mentores e interactuar con los alumnos; 2) reunirse con los estudiantes para conocer sus intereses y opiniones acerca del reto a solucionar; 3 ) tener una escucha activa y orientarlos sobre la solución que ellos propongan; 4) participar en el foro de mentoría de su equipo de trabajo; 5) utilizar los recursos de sala de chat 
y videoconferencia para tomar decisiones; 6) establecer un plan de trabajo con reuniones productivas en las que se definan previamente los temas de conversación; 7) documentar la información para que al finalizar la actividad se evalúen los resultados; 8) trabajar junto con los alumnos en la presentación final.

Durante las primeras dos semanas se les presentaron a los alumnos contenidos relacionados a los temas de emprendimiento e innovación, también se les presentaron diversas problemáticas de su entorno. Como actividades a los alumnos se les solicitó: 1) que participaran en el foro de discusión donde se presentaron y dieron respuesta a una pregunta sobre emprendimiento; 2) de las problemáticas se les pidió seleccionar la que más les interesara, participando en otro foro de discusión; los temas que eligieron los estudiantes estaban relacionados con: reducción de la pobreza, facilitar la apertura de negocios, la seguridad alimentaria, la seguridad energética, mejorar la calidad del aire y las causas de la corrupción; 3) se conformaron los grupos y se asignó un mentor que estuviera interesado en la temática; 4) comunicarse con el mentor y ponerse de acuerdo con los pasos para llegar a la solución; 5) participar colaborativamente y realizar una presentación con los resultados de su trabajo.

\section{Organización del trabajo}

Inicialmente se envió mensajes a los integrantes de cada grupo por correo electrónico para que tuvieran los datos de los mentores y pudieran comenzar a organizarse. También se formaron grupos en WhatsApp por cada problemática para que empezaran a elaborar sus propuestas de solución. El grupo de 20 estudiantes se dividió en cinco grupos, uno por mentor para que realizaran las actividades propuestas. En la plataforma en línea también se encontraba un foro para que aportaran a la solución a través de una lluvia de ideas, se solicitaba que tanto los mentores como los alumnos realizaran aportaciones a cada temática.

\section{Plataforma en línea}

Para realizar el curso se utilizó la plataforma Moodle que es un entorno de aprendizaje que utiliza el Internet para permitir que los estudiantes estén conectados de una manera flexible e innovadora. Las características de este sistema de gestión de aprendizaje (LMS por sus siglas en inglés) han favorecido que sea utilizada en diferentes niveles educativos debido a su flexibilidad y constante actualización, que la hacen una alternativa de fácil aplicación. Esta plataforma permite integrar materiales educativos multimedia, así como herramientas que favorecen la comunicación entre los participantes y que facilitan la labor de gestión escolar del docente (Baltodano y Gómez-Zermeño, 2017). Dentro de las características de este LMS que fueron utilizadas en el curso se encuentran: 1) formato semanal para presentar el curso 2) actividades como foros, blog, cuestionarios y tareas; 3 ) los alumnos pudieron marcar su avance cada vez que terminaban una actividad, lo que permitió dar seguimiento a su progreso; 4) también pudieron cargar sus propias tareas y recibir comentarios de los profesores por medio del correo electrónico; 5) incluyó otros recursos como videoconferencias y chats.

\section{Interacciones entre los participantes}

En el curso en línea los participantes tuvieron la oportunidad de interactuar en diferentes foros de discusión dentro de la plataforma, también utilizaron otras plataformas como WhatsApp y Skype. Los resultados que se presentan proceden de la matriz de observación. Se pudo observar que los mentores fueron los que tomaron la iniciativa para comunicarse con los alumnos a través del correo electrónico y los foros. Las principales propuestas fueron realizar sesiones por Skype, principalmente porque los mentores no se encontraban en el mismo lugar que los alumnos. Los ritmos de trabajo al principio fueron en su mayoría lentos en los foros, debido a la poca participación de los estudiantes. Posteriormente el ritmo de trabajo cambió al crearse grupos de trabajo en WhatsApp y reuniones por videoconferencia, también se registraron los avances en documentos de Google Drive.

En el curso se incluyeron cinco foros de discusión para que los alumnos pudieran realizar sus participaciones de manera individual y grupal. En el foro 1 la actividad a realizar consistió en la presentación de los alumnos y responder a una pregunta relacionada al emprendimiento e innovación de manera individual. En esta actividad los alumnos participaron activamente respondiendo a su interés sobre desarrollar emprendimientos, además manifestaron tener gran preocupación por los problemas sociales y compartieron la importancia del emprendimiento e innovación para el país. Por ejemplo, una de las estudiantes mencionó que ya cuenta con un emprendimiento que le puede ayudar a solucionar la problemática "el proyecto social que ya estoy emprendiendo me dio una idea muy buena para solucionar esta problemática, y es que los dos van de la mano" (Comentario de alumna en Foro 1); "sería un gusto poder desarrollar el proyecto, pues en el emprendimiento social he hallado una gran pasión" (Comentario de alumno en Foro 1); y otra estudiante comentó sobre su participación en un proyecto relacionado al tema "actualmente me encuentro en un grupo de investigación donde estamos trabajando un tema similar (Comentario de alumna en Foro 1). 
En el segundo foro los mentores tenían que presentarse y compartir sus experiencias como emprendedores, así como las principales áreas en las que se desempeñan. Ellos expresaron entusiasmo e interés por conocer los proyectos de los estudiantes. También hablaron de sus experiencias en el campo del emprendimiento, evaluación de proyectos y programas de apoyo al emprendimiento. Algunos comentarios fueron: "Estoy muy contenta de poder ser parte de este grupo de mentores. No puedo esperar a iniciar este viaje con ustedes y lograr transmitirles mis experiencias en emprendimiento para aplicarlo en su propio proyecto. Más adelante tendré el gusto de conocernos y poder platicar más a fondo quiénes somos y cómo puedo apoyarlos." (Comentario de mentora en Foro 2). "Mi mayor interés son los temas de transferencia de conocimiento, desarrollo tecnológico innovación y transferencia tecnológica, hoy me encuentro haciendo un estudio del rol de la investigación en la transferencia de conocimiento, y su impacto en el desarrollo económico y social". (Comentario de mentor en Foro 2).

En el Foro 3 correspondiente a la formación de equipos de trabajo, la participación estuvo relacionada con la escogencia de un problema a resolver para formar los grupos. Los alumnos propusieron algunas soluciones, especialmente relacionándolo con los conocimientos previos que ya tenían. También los mentores tuvieron la oportunidad de elegir el tema que les interesaba trabajar. Se formaron cinco grupos cada uno con un mentor o mentora responsable. Algunos comentarios de los estudiantes se señalan a continuación: "iHola! Me gustaría participar en este reto, ya que sería una manera de aportar al estado a lograr el cumplimiento los ODS 1 y 2, propuestos por la ONU, y que forman parte del Plan Estratégico para el Estado de Nuevo León 2015-2030. De manera que, al cumplir la meta de erradicar la pobreza extrema alimentaria en el estado de Nuevo León, la vida de 160,255 personas que actualmente se encuentran en condiciones de extrema pobreza y sin seguridad alimentaria se verá impactada positivamente." (Comentario de alumna en Foro 3). "Me interesa participar en este tema, creo que lo primordial debería ser ofrecer condiciones adecuadas para ocupar puestos de trabajo acordes con las características del puesto, así como el perfil del participante en el de tal forma que los lugares estén ocupados por personas capacitadas, y que además reciban remuneraciones adecuadas por su trabajo". (Comentario de mentor en Foro 3).

En el foro 4 se pidió realizar una actividad de lluvia de ideas. Los participantes propusieron crear grupos de WhatsApp donde pudieran ponerse de acuerdo sobre las soluciones y discutir los problemas, por lo que usaron el foro para poder sus acuerdos finales y enlaces a documentos en Google Drive donde desarrollaron sus soluciones. Los equipos que tuvieron más interacción en los foros fueron el 2 y el 5 . También utilizaron el foro para resumir los acuerdos de las juntas con el mentor, como es el caso del Equipo 4 y 6 . La participación de los mentores consistió en este foro en realizar algunas consultas sobre cómo las propuestas de los alumnos se pueden diferenciar de lo que ya existe y también buscaron orientar a los alumnos hacia la discusión de la problemática. Por ejemplo: "actualmente se da mucho la publicidad en las redes sociales y ya hay muchas páginas donde todo mundo inclusive promociona sus servicios, ¿cuál es la diferencia?” Mentora Equipo 4. También el mentor del Equipo 5 comentó en el foro.

"Creo que la idea es buena, pero en realidad hoy en día muchos de esos trámites ya se llevan a cabo en línea, algunos, otros no, mi pregunta es, ¿en realidad esto ha disminuido los actos de corrupción que se presentan? "(Comentario de mentor en Foro 4). El último foro correspondió a la despedida del curso, en el que los alumnos y mentores dejaron mensajes sobre su experiencia en el curso. Uno de los comentarios se presenta a continuación. "Muchas gracias por el apoyo, fue un placer tomar este taller con usted. Siempre muy atenta con nosotros y al pendiente de los comentarios del taller. Y por otro lado me gustaría agradecerle a mi mentora, por su tiempo y sus sugerencias que me permitieron ampliar mi perspectiva y hacer más efectiva mi forma de comunicación, observando cosas que no había notado. iMuchas felicidades por su compromiso y dedicación!" (Comentario de alumna foro 5).

La Figura 2 muestra la cantidad de participaciones en los foros, en total fueron 128 interacciones. El foro que tuvo mayor interacción fue el de presentación de los alumnos (58 interacciones), seguido por el de formación de equipos de trabajo (35 participaciones). Una vez que los alumnos tenían que aportar de manera colaborativa en la lluvia de ideas, la participación se volvió lenta y no se estaba llegando a los acuerdos que se requería en el tiempo establecido, por lo que se crearon grupos en WhatsApp para permitir que los alumnos pudieran contactarse más rápidamente con los mentores.

En esta experiencia la mayor participación se dio en los grupos de WhatsApp donde se organizaron grupos de trabajo para ponerse de acuerdo en la forma de llevar a cabo la solución. Los resultados de las conversaciones se dividieron por equipo. Aquí la cantidad de participaciones fue muy superior a la de los foros, en total se presentaron 553 interacciones. En la Tabla 2 se presentan las principales interacciones en esta herramienta. En este estudio los participantes utilizaron en su mayoría la herramienta para llegar a acuerdos sobre las reuniones y las propuestas de solución, luego trabajaban en los foros o en documentos de Google Drive, que fueron elaborados por ellos, donde colocaban los resultados. En la Figura 3 se muestran las interacciones en WhatsApp por cada equipo, siendo las de mayor cantidad los equipos 4 y 5 y la de menor cantidad el equipo 1. 


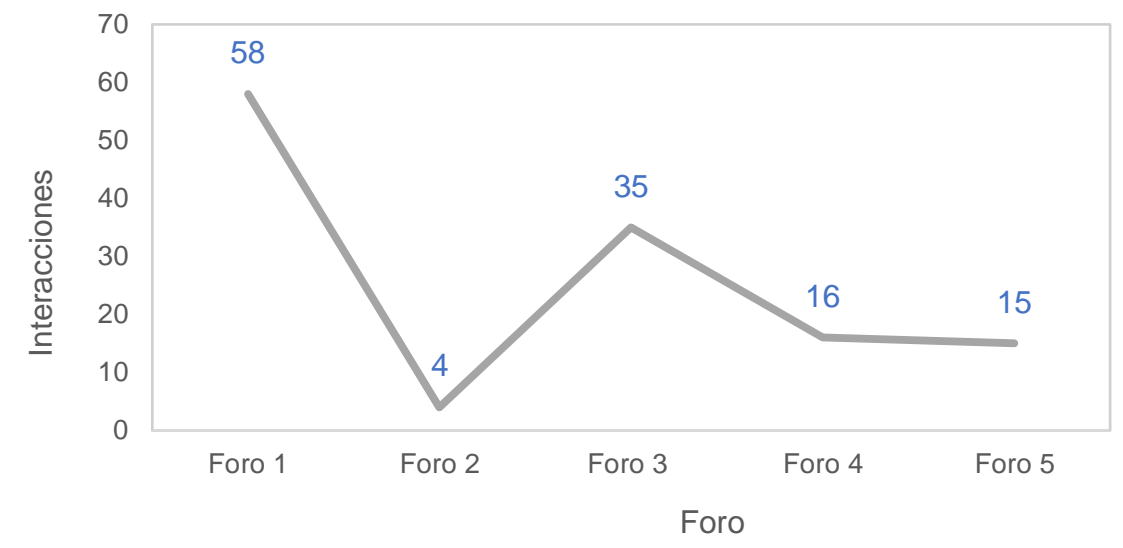

Fig. 2: Participaciones en los foros de discusión. a) Foro 1. Presentación de alumnos, b) Foro 2. Presentación de mentores, c) Foro 3. Formación de equipos de trabajo, d) Foro 4. Lluvia de ideas, e) Foro 5. Despedida

Tabla 2: Matriz de observación en los grupos de WhatsApp

\begin{tabular}{|c|c|c|}
\hline $\begin{array}{l}\text { Temática } \\
\text { abordada }\end{array}$ & Equipo & Resultados observados \\
\hline $\begin{array}{l}\text { Seguridad } \\
\text { energética }\end{array}$ & 1 & $\begin{array}{l}\text { Ese equipo fue el que menos trabajó con el mentor. El mentor tomó la iniciativa para motivar la } \\
\text { participación presentando un enlace a un podcast sobre la problemática. Luego los alumnos } \\
\text { realizaron dos propuestas de solución que fueron retroalimentadas por el mentor para orientarlos } \\
\text { hacia una solución, pero los alumnos presentaron el producto final sin la participación del mentor. }\end{array}$ \\
\hline $\begin{array}{l}\text { Facilitar la } \\
\text { apertura de } \\
\text { negocios }\end{array}$ & 2 & $\begin{array}{l}\text { Lo utilizaron para presentar sus ideas y para compartir opiniones entre los miembros del grupo y } \\
\text { la mentora quien les dio sugerencias para buscar validación de la idea. También se pusieron de } \\
\text { acuerdo para reunirse por Skype y revisar la presentación final con la mentora que los } \\
\text { retroalimentó sobre la propuesta. }\end{array}$ \\
\hline $\begin{array}{l}\text { Causas de } \\
\text { la corrupción }\end{array}$ & 3 & $\begin{array}{l}\text { Los alumnos comenzaron a presentar sus ideas y el mentor hizo consultas sobre la viabilidad de } \\
\text { la propuesta. Propusieron ir colocando las propuestas en Google drive para revisarlas y contribuir } \\
\text { a las ideas. Al finalizar la actividad presentaron el trabajo al mentor para que los retroalimentara. }\end{array}$ \\
\hline $\begin{array}{l}\text { Reducción } \\
\text { de la } \\
\text { pobreza }\end{array}$ & 4 & $\begin{array}{l}\text { Fueron las que más participaciones tuvieron en el WhatsApp y quienes tuvieron interacción } \\
\text { durante todo el tiempo de la actividad. Utilizaron la herramienta principalmente para coordinar } \\
\text { reuniones convocadas por la mentora. Las alumnas propusieron utilizar Google docs para realizar } \\
\text { la tormenta de ideas y lo colocaron en el foro. La mentora compartió enlaces con datos sobre las } \\
\text { problemáticas. Alumnas compartieron los resultados de su proyecto: propuesta y validación, } \\
\text { también compartieron la presentación final para que la mentora les pudiera dar retroalimentación. }\end{array}$ \\
\hline $\begin{array}{l}\text { Mejorar la } \\
\text { calidad del } \\
\text { aire }\end{array}$ & 5 & $\begin{array}{l}\text { La mentora comenzó la participación y las alumnas presentaron una investigación que hicieron } \\
\text { sobre el tema. También se pusieron de acuerdo para tener una junta vía Skype y llegaron a } \\
\text { acuerdos para dar forma a la idea, las alumnas trabajaron la propuesta y se la presentaron a la } \\
\text { mentora quien les dio retroalimentación. En esta etapa utilizaron el WhatsApp para afinar los } \\
\text { detalles de la propuesta, compartieron documentos en Google Drive relacionados con } \\
\text { regulaciones y revisaron la presentación final. }\end{array}$ \\
\hline
\end{tabular}

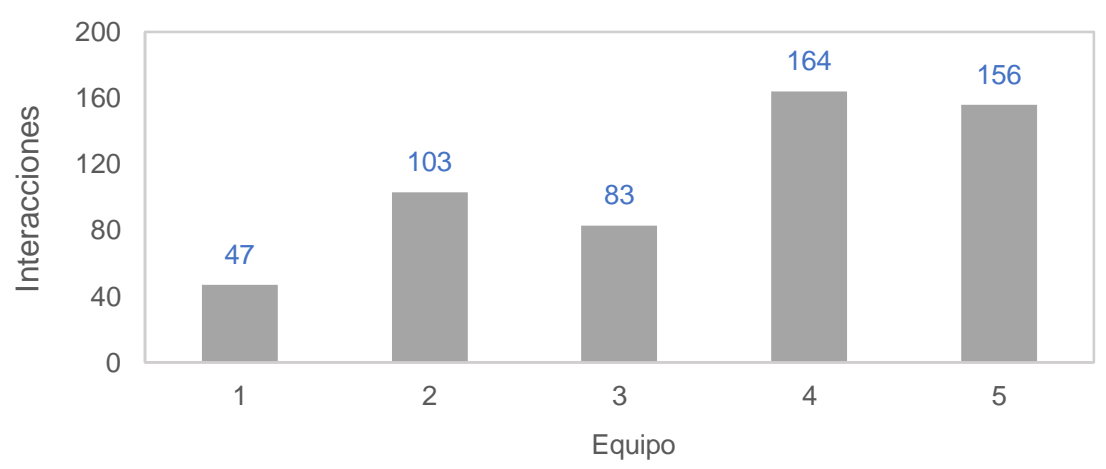

Fig. 3: Interacciones en WhatsApp

También se utilizó Skype como herramienta para realizar videollamadas, donde los mentores dieron las pautas para orientar el trabajo de los estudiantes hacia el desarrollo de las propuestas. Tres de los cinco equipos utilizaron este medio para reunirse con los mentores y definir la estrategia de solución. 


\section{Elementos de la mentoría observados en la experiencia}

En la experiencia de la mentoría se presentaron los siguientes elementos: 1) los mentores buscaron orientar a los alumnos sobre sus ideas, analizando si eran relevantes para solucionar la problemática escogida; 2) los mentores buscaron hacer preguntas que llevaran a la reflexión de las soluciones; 3 ) recomendaron buscar opiniones de posibles usuarios o interesados en sus propuestas para validar la idea, para que las propuestas estuvieran basadas en datos y no en creencias de las soluciones; 4) también existió disponibilidad de los mentores para reunirse con los alumnos en diferentes horarios y medios; 5) los mentores retroalimentaron a los alumnos en su presentación final.

En el caso de los alumnos que trabajaron con el mentor, los elementos presentados fueron: 1) acordaron reuniones con los mentores para presentar sus ideas; 2) buscaron más información sobre las propuestas de solución; 3) documentaron los resultados obtenidos con la problemática; 4) discutieron con el mentor los hallazgos; 5) reflexionaron sobre la viabilidad de las propuestas; 5) eligieron y desarrollaron la que consideraron más conveniente y 6 ) recibieron retroalimentación de su presentación final. De acuerdo con lo observado durante el trabajo de los mentores y los estudiantes, los participantes siguieron los pasos que se muestran en la Tabla 3.

Los principales problemas se dieron para coordinar las reuniones con los estudiantes por lo que algunos alumnos no continuaban con la comunicación debido a problemas de horario. Aunque en la mayoría de los casos se realizaron las actividades, no siempre se involucró a los mentores o solamente se les presentó la propuesta cuando ya la tenían finalizada para recibir retroalimentación y no siguiendo el proceso que se esperaba de seguimiento durante toda la actividad. También faltó formular un plan de trabajo por los mentores previo a las reuniones. En los casos que sí se trabajó con el mentor, se pudo llegar a soluciones mejor sustentadas y basadas en la investigación y la reflexión de la problemática.

Tabla 3: Actividades realizadas en la mentoría para llegar a una propuesta de solución

\begin{tabular}{|l|l|}
\hline Fases & Actividades \\
\hline Definir la problemática a resolver & ¿Cuál es el problema a resolver? Definir el tema. \\
\hline Identificar la población & ¿Qué territorio vamos a cubrir: urbano, rural, ¿condiciones climáticas? \\
& ¿Cómo funciona el sistema? \\
& Determinar la población que tiene el problema y buscar datos. \\
\hline Vincular el problema con las causas & $\begin{array}{l}\text { Socioeconómicos, conductas, culturales. } \\
\text { Definir el grupo sociodemográfico que se quiere atender: elegir un } \\
\text { municipio. }\end{array}$ \\
\hline Validar la propuesta de solución & Cuantificar el tamaño de la población: escalabilidad \\
\hline Propuesta & $\begin{array}{l}\text { Hacer supuestos } \\
\text { Validarlos con quienes están viviendo la problemática }\end{array}$ \\
& Proponer una solución \\
\hline & $\begin{array}{l}\text { Programa social: educativo, prestar bien o servicio } \\
\text { Producto desde el sector privado } \\
\text { Alianza público-privada } \\
\text { Política pública }\end{array}$ \\
\hline
\end{tabular}

Reportes del mentor y alumnos sobre los resultados de la experiencia educativa

A continuación, se presentan las respuestas a las preguntas relacionadas con la mentoría realizadas a los mentores y alumnos por equipo.

\section{Reporte del mentor}

A la pregunta sobre ¿en qué consistió la mentoría? Para el mentor del equipo 1 la mentoría consistió en su disposición para atender preguntas, dudas u observaciones de los alumnos. Para el mentor del equipo 2, la mentoría realizada consistió principalmente en la preparación y evaluación de una idea de negocio para realizar una presentación a una audiencia. Para el mentor del equipo 3 consistió en dar asesoría y realizar una lluvia de ideas y para los mentores de los equipos 4 y 5 , guiar a los alumnos en su proyecto de emprendimiento, así como dar sesiones de trabajo para guiar el trabajo e intereses de los estudiantes.

A la pregunta sobre ¿cuáles fueron los aprendizajes de los alumnos?, el mentor del equipo 1 respondió que no lo sabía porque su interacción fue mínima "...logramos intercambiar unos breves mensajes a través del Whats, pero nunca recibí de parte de los participantes ninguna pregunta específica o duda respecto al tema de energías renovables, aunque les presenté unos videos sobre el tema". La mentora del equipo 2 comentó que los alumnos aprendieron sobre el trabajo que hay detrás de la presentación de una idea de negocio robusta; el mentor del equipo 3 que aprendieron a identificar sus habilidades de emprendimiento. La mentora 
del equipo 4 respondió que los aprendizajes fueron cómo realizar una presentación y herramientas de trabajo para comprender mejor el problema que desean atender. La mentora del Equipo 5 no consideró que los alumnos tomaran sus recomendaciones en cuenta en la presentación final.

Sobre la pregunta de ¿cuál es su opinión sobre el trabajo de los alumnos? El mentor del equipo 1 respondió que no los conoció lo suficiente. La mentora del equipo 2 consideró al WhatsApp como una herramienta de comunicación apropiada para aumentar la comunicación, aunque faltó motivación por parte de los estudiantes. Dentro de los equipos existieron dos actitudes de los alumnos, por un lado, estaban quienes estuvieron comprometidos con las actividades y el curso y motivados a aprender y a seguir las recomendaciones de los mentores. Por otro lado, se encontraron alumnos poco comprometidos con el curso, por lo que las interacciones con el mentor fueron difíciles de alcanzar. Por ejemplo, la mentora del equipo 4 comentó "las alumnas muy aplicadas y con mucho interés para su asignatura"; mientras que la mentora del equipo 5 indicó: "los alumnos disponen de muy poco tiempo para realizar las actividades y poco compromiso con el curso, haciendo muy complicada la interacción con ellos y disminuyendo la calidad de lo que presentaban".

A la pregunta de ¿cuáles fueron los obstáculos que se presentaron? los mentores de los equipos 4 y 5 mencionaron principalmente el tiempo, el mentor del equipo 2 la apatía de algunos alumnos, y los mentores del equipo 1 y 3 , la falta de ideas y poca proactividad de los estudiantes que afectó que se presentaron mejores propuestas. Para mejorar las propuestas, los mentores de los equipos 4 y 5 recomiendan dar mayor tiempo para realizar las actividades, la mentora del equipo 2 recomendó diseñar una metodología que los guíe paso a paso para hacer una presentación a través de una aplicación. Los mentores de los equipos 1 y 3 realizar actividades de forma presencial y la mentora del equipo 4 tener una agenda más estricta para las juntas (que obligatorias y con entregables claros). Así como poder dar seguimiento a la idea después del curso para conocer si la mentoría les ayudó a mejorarlas.

\section{Reporte de los alumnos}

A la pregunta sobre ¿qué aprendí del mentor que participó en el curso? Los alumnos del equipo 1 mencionaron que pudieron aprender sobre nuevos tipos de energía que pueden ser potenciales, pero que por el poco tiempo disponible no pudieron interactuar más, uno de ellos comentó "aprendí muy poco por el tiempo del que dispusimos, me hubiera gustado participar más con el mentor" (Equipo 1). Para los alumnos del equipo 2, los aprendizajes estuvieron relacionados con la importancia de la comunicación efectiva, así como "que es necesario escuchar diferentes perspectivas de una idea para poder mejorarla" (Equipo 2). Los alumnos del equipo 3 respondieron que aprendieron a realizar una investigación sobre productos similares al suyo y recibieron comentarios para mejorar la idea. Una de ellas comentó: "el mentor asignado siempre fue muy atento con nosotras y aprendí que es importante reconocer el "dolor" del problema y de la gente para poder aliviarlo con nuestras propuestas" (Equipo 3).

En cuanto al Equipo 4 consideraron que el apoyo de la mentora fue esencial para lograr los objetivos propuestos, así como llegar a una propuesta de solución viable. Como comentaron: "nuestra mentora nos ayudó muchísimo siendo una guía en la metodología para poder estructurar todo el proceso y llegar a una propuesta de solución realista, factible y bien validada" (Equipo 4). Para el equipo 5, la mentora les dio consejos sobre lo necesario para realizar la presentación tomando en cuenta a quién va dirigida, así como trabajar en la idea para que no se desvanezca: "La mentora nos dio muchos consejos sobre todo lo que se necesita para presentar la idea, y que es muy importante a quién va dirigido debido a que debes enfocar tu proyecto hacia el círculo en el que se desenvuelve la persona o empresa a la cual le presentarás tu proyecto" (Equipo 5).

Sobre la pregunta de ¿cuáles problemas enfrentaron y cómo los manejaron? los alumnos de los cinco equipos coincidieron en que el tiempo y sus actividades académicas fueron obstáculos para realizar las actividades. El equipo 1 indicó que su principal fue el tiempo para trabajar el curso; en el equipo 2 lo solventaron siendo flexibles ante nuevas opiniones; el equipo 3 buscó organizar el tiempo para presentar la propuesta, así como el equipo 4 que gracias al trabajo en equipo pudieron resolver el reto y llegar a la solución; por último, el equipo 5 buscó diferentes propuestas y eligieron la que mejor solucionara el problema.

Para la pregunta ¿qué les gustó más del curso y qué menos? El equipo 1 respondió que las experiencias en el curso fueron buenas: "Considero que en general las experiencias que tuve en el curso fueron buenas" Se sintió bien presentar la idea ante un público y vivir la experiencia del emprendimiento" (Equipo 1). Lo que no les gustó fue la falta de tiempo. Para el equipo 2 lo importante fue el intercambio de opiniones y la retroalimentación, también mencionaron la falta de tiempo. En el equipo 3 las alumnas indicaron haber aprendido sobre sus habilidades de emprendimiento, así como conocer las ideas de sus compañeros: "La experiencia de conocer las ideas de emprendimiento de mis compañeros y saber sus motivaciones por entrar al curso. Creo que todos tenemos la misma motivación y deseo de emprender"; comentaron que les hubiera gustado tener sesiones presenciales para conocer con quién iban a trabajar y poder visitar ejemplos de emprendimientos exitosos. 
Para el equipo 4 lo mejor fue la colaboración en el equipo y la mentoría; sin embargo, una de ellas consideró la plataforma algo confusa, así como el tiempo para realizar todas las actividades para llegar a la propuesta de solución y presentarlas. El equipo 5 indicó que les gustó el trabajo en equipo y con la mentora, de esta interacción surgieron ideas para su propuesta: "trabajar en equipo, con mi compañera y la mentora, debido a las ideas que surgieron de las tres, me gustó mucho el enfoque que le dimos al proyecto" (Equipo 4) y el tiempo fue mencionado como un factor negativo para este grupo.

\section{DISCUSIÓN}

A partir de la información generada durante el curso en línea y el análisis de los resultados obtenidos se pudo dar respuesta a las preguntas críticas identificadas en la sistematización del proceso de mentoría realizado. En esta etapa se presentan las relaciones, principios y alcance de los resultados; así como la existencia de puntos inciertos y las implicaciones de esta investigación, comparando los resultados obtenidos con la literatura existente.

En primer lugar, se pudo observar que los ritmos de trabajo de los alumnos fueron rápidos al principio, especialmente cuando las tareas se realizaban de forma individual. Sin embargo, comenzaron a tener problemas para trabajar dentro de la plataforma en los foros de lluvia de ideas, donde se esperaba un trabajo colaborativo. El uso de la herramienta de WhatsApp favoreció que las interacciones fueran más rápidas y se incrementara la participación y las interacciones entre los estudiantes y los mentores. Esto fue similar para la mayoría de los grupos que se comunicaron con esta herramienta. El uso de foros en la plataforma no favoreció la interacción de los participantes, el curso se activó cuando se les dio la oportunidad de utilizar el WhatsApp como medio de comunicación.

El chat y videoconferencia dentro de la plataforma no se utilizaron, más bien la elección fue realizar las reuniones en Skype y compartir documentos en Google Drive. Estos resultados concuerdan con otros estudios. En cuanto al uso de Whatsapp esta herramienta ha incrementado su uso en los últimos años en los cursos en línea debido a que facilita la interacción entre estudiantes y docentes (Robles et al., 2019). Además de que permite monitorear actividades de aprendizaje colaborativo, así como compartir información; por otro lado, cuando es necesario integrar otros documentos o herramientas de colaboración se utilizan otras herramientas en línea como Google Drive (Annamalai, 2019).

El surgimiento de nuevas formas de comunicación a través de aplicaciones móviles, han incrementado la oportunidad de que los estudiantes puedan interactuar con sus compañeros y profesores en tiempo real y en cualquier momento y lugar (Robles et al., 2019). Por lo que esa forma de recibir respuesta inmediata fue más eficiente, en este caso, que la participación en los foros que han sido utilizados tradicionalmente en la educación en línea para fomentar la interacción y el trabajo colaborativo en los estudiantes. Se considera que este hallazgo indica que las universidades se enfrentan a un cambio de paradigma, en el que el reto es facilitar vías de comunicación que sean más eficientes para alcanzar los objetivos educativos y la motivación de los estudiantes para aumentar su participación en esta modalidad a distancia.

Al analizar las respuestas de mentores y alumnos, se encuentra que en el equipo 1 el mentor tuvo disposición de compartir, pero los alumnos no tuvieron interés en acercarse, por lo que no existió colaboración entre ellos. Los alumnos lo atribuyeron al tiempo, pero indicaron haber querido conocer más al mentor y que aprendieron de él sobre las energías. Aunque tuvieron las oportunidades para interactuar y la disposición del mentor, los estudiantes no lo hicieron. El equipo 2 sí tuvo oportunidad de interactuar, la mentora consideró que faltó motivación de los alumnos, los estudiantes utilizaron la mentoría más para recibir retroalimentación de lo que habían hecho, que de trabajar junto a la mentora. En el equipo 3 existió mayor interacción con el mentor y las estudiantes siguieron las pautas para identificar el problema y llegar a la solución, también recibieron retroalimentación del mentor sobre la presentación final.

El equipo 4 fue el que más tuvo interacción con la mentora, tuvieron al menos cuatro juntas por Skype, realizaron entrevistas y documentaron evidencias durante todo el periodo, las opiniones de la mentora y las estudiantes fueron favorables hacia el resultado de la mentoría. Siendo importantes el interés del mentor y la disciplina del alumno para lograr los resultados. Por último, para la mentora del equipo 5 aunque considera que los estudiantes no tomaron en cuenta sus apreciaciones, las alumnas sí mencionaron esos aprendizajes para su presentación final.

Se encontró que dos de los grupos presentaron la solución en el tiempo límite, por lo que tuvieron poco tiempo para trabajar con los mentores en toda la propuesta (Equipos 1 y 2), los otros tres trabajaron en varias sesiones de mentoría que les permitió realizar una solución más estructurada y analizada. En el caso de los alumnos que no planificaron bien su tiempo, por lo que no avanzaron según lo estipulado, pudo haber incidido el que el curso es opcional y los estudiantes dieron prioridad a sus materias curriculares. Se pudo observar en dos estudiantes poco interés en reunirse con el mentor, a pesar de que ésta era una persona muy experimentada, los alumnos prefirieron realizar el trabajo por su cuenta. Esto concuerda con los resultados 
presentados en Ting et al. (2017), en los que cuando la comunicación no fue efectiva no se logró establecer una relación entre mentor y aprendiz y no se dieron los resultados esperados.

Existieron tres casos exitosos en los que los participantes pudieron realizar las actividades solicitadas, se destaca el equipo 4 que, gracias al interés de las alumnas hacia el curso, aunque tenían una carga académica alta también cumplieron con las actividades propuestas y se mantuvieron constantemente trabajando junto con su mentora desde el principio. En estos casos se pudo observar que se obtuvieron algunos beneficios de la mentoría indicados por Babatunde y El-Gohari (2019) como fueron: el acceso a consejos prácticos, aprender de la experiencia de otros y favorecer el autoaprendizaje cuando se promovieron actividades de investigación de las problemáticas y de las formas de solucionarlas.

En los casos en que participaron los mentores, la orientación fue similar en cuanto a los pasos para llegar a una solución, lo que puede deberse a que los mentores tenían una formación similar en materia de creación de emprendimientos y también que tuvieran experiencia previa para buscar soluciones. En este caso, a los mentores sólo se les dieron indicaciones sobre la metodología del curso y las actividades a realizar, pero como se mencionó anteriormente la manera de desarrollar las propuestas la haría cada uno según la necesidad del tema de estudio y las posibilidades. Por lo anterior se considera que la forma de resolver los problemas coincidió con los pasos seguidos por Sendouwa et. al (2019) y Nabi et al. (2018) donde se definió un problema con posibles soluciones, se analizan a través de diversas técnicas y se elige la que consideran más apropiadas con un rol activo de los estudiantes, quienes son los que buscan la solución guiados por los mentores. Los pasos recomendados para realizar la mentoría se presentan en la Figura 4.

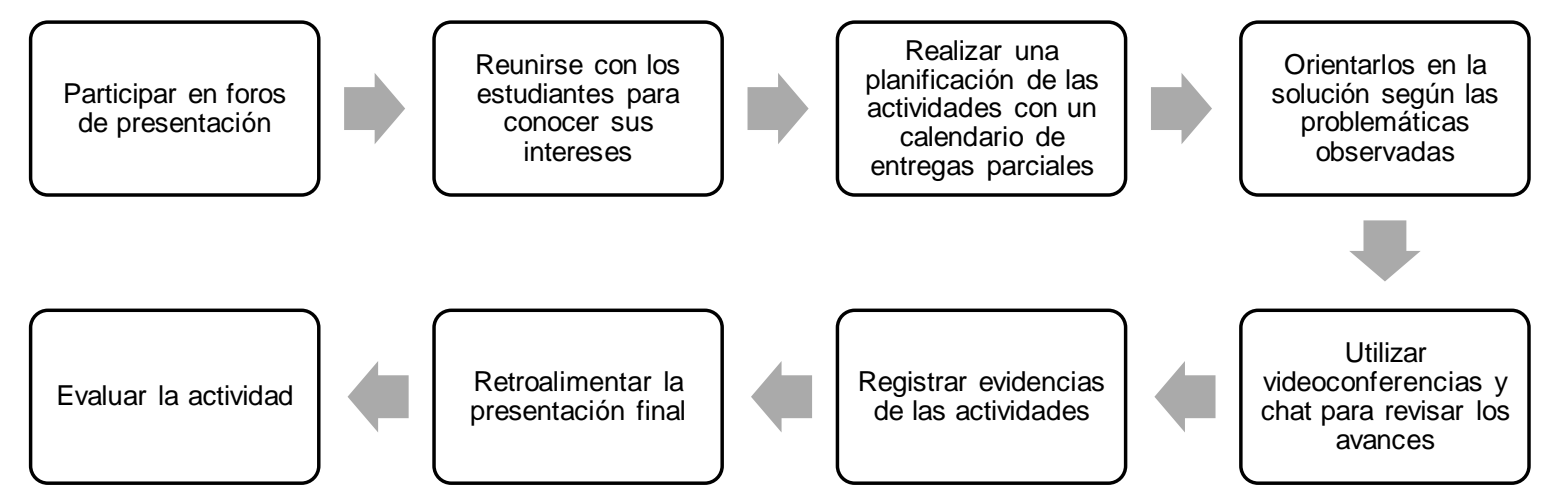

Fig. 4: Pasos a seguir en una mentoría de un curso en línea en emprendimiento

Las problemáticas presentadas en el curso fueron de interés de la mayoría de los alumnos, sin embargo, uno de ellos manifestó que esos temas sociales no son de su interés por lo que prefirió no participar en la solución, por lo que es necesario continuar investigando en los casos de los estudiantes que no tengan este interés para proponer otro tipo de actividades (Bell y Bell, 2016). En el perfil de alumno se puede ver que al hacer la consulta de si les gustaría emprender, la mayoría tiene mayor inclinación hacia solucionar problemas de la comunidad. Debido a que tienen un gran conocimiento técnico, las propuestas presentadas se relacionaron con sus carreras. Al finalizar el curso, la intención emprendedora se mantuvo y los alumnos manifestaron estar satisfechos con el resultado de la mentoría. Esto puede explicarse por las razones que brindan Nabi et al. (2019) y Sharafizad (2017) en la que mencionan que el modelado de roles realizado en la mentoría les permitió visualizar la idea del emprendimiento y fortalecer la idea de que es factible realizarlo manteniendo las intenciones de emprender.

En el caso de la disposición del alumno a compartir con el mentor, se encontró que no se dio una sinergia en todos los casos, por lo que se recomienda seguir indagando en estas razones. Estos resultados se oponen a los que mencionan Ting et al., 2017; quienes establecen que además de crear un grupo de mentores de calidad, es importante estimular el entusiasmo de los mentores. En este caso los mentores sí tuvieron intención de colaborar con los estudiantes. Una contradicción que se presentó fue con los alumnos que no participaron con el mentor, aunque tuvieron la oportunidad de reunirse no lo hicieron y luego mencionaron que les hubiera gustado conocerlo más. Estos mismos autores concluyen que existe un "desajuste en la mentoría", cuando los jóvenes emprendedores que nunca han recibido educación emprendedora demuestran una capacidad de absorción y una intención de aprendizaje débil (Ting et al., 2017). Esto también es contradictorio, debido a que la universidad en que se desarrolló el estudio cuenta con un ecosistema emprendedor con gran cantidad de opciones para formar en emprendimiento, sin embargo, la mayoría de las estudiantes que participaron de este estudio no habían llevado ningún curso o solamente uno durante toda su carrera. 
Dentro de las limitaciones del estudio se encuentra que los resultados son propios del grupo de estudio analizado, sin embargo, podría ser replicado en otros contextos para evaluar en profundidad los factores de éxito o fracaso de la mentoría en cursos en línea. Las aportaciones de esta investigación están relacionadas con la presentación de los resultados de un estudio empírico sobre mentoría en cursos de emprendimiento en línea en educación superior. La cual viene a cubrir en parte la falta de este tipo de estudios (Tinoco-Giraldo et al., 2020), presentando algunos principios de diseño que pueden ser utilizados en otros cursos o contextos, según la experiencia sistematizada en este estudio. Debido a que la sistematización de experiencias pone énfasis en el proceso vivido durante la experiencia concreta y busca describir y entender qué es lo que sucedió y por qué ocurrió, en este estudio se buscó poder explicar cómo vivieron los participantes este proceso y extraer lecciones que permitan mejorarlos en una futura experiencia (Barbosa-Chacón et al., 2015; InciarteGonzález et al. 2017; Jara, 2018; Picado-Alfaro, 2018). Por lo tanto, el realizar esta sistematización permitió conocer a fondo las actividades llevadas a cabo por los participantes y poder extraer elementos propios de la experiencia vivida durante el proceso de mentoría, los pasos que se siguieron para alcanzar los objetivos, así como los obstáculos enfrentados y las opiniones de los participantes.

También llama la atención sobre la disposición de los estudiantes a aprender sobre emprendimiento dentro de un ecosistema emprendedor universitario, en que se considera necesario continuar indagando sobre el aprovechamiento que hacen los alumnos de los cursos extracurriculares y curriculares que en esta materia se realizan en esta universidad para conocer su impacto y la manera de obtener una mayor participación y posteriormente conocer cómo utilizan esos conocimientos adquiridos (Caban et al., 2018; Herrera y Villalobos, 2018). Por último, advierte sobre las nuevas formas de comunicación en los ambientes virtuales de aprendizaje, para reconocer cuáles son los más efectivos y generar mayor interacción entre alumnos y maestros, así también los que favorezcan el interés y motivación en el proceso de enseñanza-aprendizaje (Robles et al., 2019), especialmente en la coyuntura que se vive actualmente.

\section{CONCLUSIONES}

El objetivo planteado para esta investigación buscaba analizar los elementos que intervinieron en la mentoría de un curso en línea de emprendimiento con estudiantes universitarios de carreras profesionales, a través de una sistematización de la experiencia educativa para poder ser replicada en otros cursos. Según el análisis presentado se pueden extraer las siguientes tres conclusiones principales: 1) Los elementos de la mentoría que se presentaron en esta experiencia fueron: comunicación entre alumnos y mentores para definir los pasos a seguir hacia lograr una solución al problema planteado utilizando diferentes medios de comunicación en línea; guía del mentor para que los alumnos reflexionaran sobre la viabilidad de sus propuestas y retroalimentación para mejorar las presentaciones finales. 2) Para replicar esta experiencia en otros cursos se pueden seguir las actividades descritas en esta investigación, así como los pasos sugeridos en este estudio, mitigando los obstáculos de tiempo y de comunicación que tuvieron los participantes a través de una planificación con un calendario de entregas para mejorar los resultados obtenidos. 3) Considerar que existe un cambio de paradigma del uso de herramientas tradicionales en los cursos en línea como son los foros y utilizar otras herramientas de comunicación que permitan generar mayor interacción entre los participantes.

\section{AGRADECIMIENTOS}

Los autores desean agradecer el apoyo financiero de Writing Lab, TecLabs, Tecnologico de Monterrey, México, en la producción de este trabajo. Además, agradecen al fondo Novus 2019 del Tecnologico de Monterrey.

\section{REFERENCIAS}

Annamalai, N., Using WhatsApp to Extend Learning in a Blended Classroom Environment, Teaching English with Tech., ISSN: 1642-1027, 19(1), 3-20 (2019).

Babatunde, S. y El-Gohary, H., Necessity of Mentoring in Entrepreneurship Education: Reflection by Practitioners, doi: 10.1061/(asce)ei.1943-5541.0000399, J. Prof. Issues Eng. Educ. Pract., 145(1), 1-5 (2019).

Baltodano, M. y Gómez-Zermeño, M.G., Pedagogical, curricular and didactic element involved in the creation of an elearning environment: the case of a Costa Rican university, https://doi.org/10.17718/tojde.340396, Turkish Online Journal of Distance Education, 18(4), 104-118 (2017).

Barbosa-Chacón, J.W., Barbosa, J.C. y Rodríguez, M., Concepto, Enfoque y Justificación de la Sistematización de Experiencias Educativas una Mirada "Desde" y "Para" El Contexto de la Formación Universitaria, doi: 10.22201/iisue.24486167e.2015.149.53128, Perfiles Educativos, 37(149), 130-149 (2015).

Bell, R. y Bell, H., An Enterprise Opportunity for Entrepreneurial Students: Student Enterprise Development and Experience Assessed Through the Student Voice, doi: 10.1108/et-12-2014-0150, Educ. + Training, 58(7/8), 751-765 (2016) 
Cabana, S. R., Cortés, F.H., Aguilera, M.I. y Vargas. F.A., Factores Determinantes para el Intraemprendimiento Social: El Caso de los Estudiantes de Ingeniería de la Universidad de La Serena, Chile, http://dx.doi.org/10.4067/S071850062018000200087, Formación Universitaria, 11(2), 87-98 (2018).

Contreras-Velásquez, J.C., Wilches-Durán, S.Y., Graterol-Rivas. M.E. y Bautista-Sandoval, M.J., Educación Superior y la Formación en Emprendimiento Interdisciplinario: Un Caso de Estudio, doi:10.4067/S0718-50062017000300003, Formación Universitaria, 10(3), 11-20 (2017).

Falkembach, E. y Torres, A., Systematization of Experiences: A Practice of Participatory Research from Latinamerica; In: The SAGE Handbook of Action Research by H. Bradbury, pp 76-82 SAGE Publications Ltd., Londres, Inglaterra (2015).

Gimmon, E., Mentoring as a Practical Training in Higher Education of Entrepreneurship, doi:10.1108/et-02-2014-0006 Educ. + Training, 56 (8/9), 814-825 (2014).

Hägg, G. y Kurczewska, A., Connecting the Dots: A Discussion on Key Concepts in Contemporary Entrepreneurship Education, doi: 10.1108/et-12-2015-0115, Educ. + Training, 58(7/8), 700-714 (2016).

Herrera, R.A. y Villalobos, M.A., Caracterización de la Formación Académica en Emprendimiento de Pregrado que se realiza en Universidades Chilenas, http://dx.doi.org/10.4067/S0718-50062018000400043, Formación Universitaria, 11(4), 43-52 (2018).

Inciarte-González, A., Camacho, H. y Casilla, D., Sistematización de Experiencias Formativas en Competencias Docentes Investigativas, Opción, ISSN: 1012-15877, 33(82), 322-343 (2017).

Jara, O., La sistematización de Experiencias. Práctica y Teoría para otros Mundos Posibles, 8-258, Centro Internacional de Educación y Desarrollo Humano CINDE, Bogotá, Colombia (2018).

Kubberød, E., Fosstenløkken, S.M. y Erstad, P.O., Peer Mentoring in Entrepreneurship Education: Towards a Role Typology, doi: 10.1108/et-08-2017-0109, Educ. + Training, 60(9),1026-1040 (2018).

Lackéus, M., Entrepreneurship in Education: What, Why, When, How, 1-45, OECD Publishing, París, Francia (2015).

Lefebvre, M.R. y Redien-Collot, R., How to Do Things with Words: The Discursive Dimension of Experiential Learning in Entrepreneurial Mentoring Dyads, doi: 10.1111/jsbm.12022, J. of Small Bus. Manag., 51(3), 370-393 (2013).

Nabi, G., Walmsley, A. y Akhtar, I., Mentoring Functions and Entrepreneur Development in the Early Years of University, doi:10.1080/03075079.2019.1665009, Studies in Higher Education, 1-16 (2019).

Nabi, G., Walmsley, A., y otros tres autores, Does Entrepreneurship Education in the First Year of Higher Education Develop Entrepreneurial Intentions? The Role of Learning and Inspiration, doi: 10.1080/03075079.2016.1177716, Stud. Hig. Educ., 43(3), 452-467 (2018).

OCDE. The Future of Education and Skills. Education 2030. OECD Publishing, Paris, Francia (2018).

Padilla-Meléndez, A., Fernández-Gámez, M.A. y Molina-Gómez, J., Feeling the Risks: Effects of the Development of Emotional Competences with Outdoor Training on the Entrepreneurial Intent of University Students, doi: 10.1007/s11365014-0310-y, Int. Entrepren. Manag. J., 10(4), 861-884 (2014).

Picado-Alfaro, M., Sugerencias Didácticas para la Implementación de un Curso de Nivelación en Matemáticas: La Sistematización de una Experiencia en la Universidad Nacional de Costa Rica, doi: 10.15359/ree.22-3.156, Revista Electrónica Educare, 22(3), 1-18 (2018).

Portuguez, M., Ross, C. y Gómez, M.G., The Impact of Higher Education on Entrepreneurship and the Innovation Ecosystem: A Case Study in Mexico, doi: 10.3390/su11205597, Sustainability, 11(20), 1-17 (2019).

Rigg, C. y O'Dwyer, B., Becoming an Entrepreneur: Researching the Role of Mentors in Identity Construction, doi: 10.1108/00400911211236181, Educ. + Training, 54(4), 319-329 (2012).

Robles, H., Guerrero, J., Llinas, H. y Montero, P., Online Teacher-Students Interactions Using WhatsApp in a Law Course, doi: 10.28945/4321, J. of Inf. Technology Educ.: Research, 18, 231-252. (2019).

Sánchez, J.C., Ward, A., Hernández, B. y Florez, J.L., Educación Emprendedora: Estado del Arte, doi: 10.20511/pyr2017.v5n2.190, Propósitos y Representaciones, 5(2), 401-473 (2017).

Scott-Kemmis, D., The role of VET in the entrepreneurial ecosystem, 1-64, NCVER, Adelaide, Australia. (2017).

Sendouwa, R.H.E., Lonto, A.L. y Saroingsong, S.J.R., Entrepreneurship Development Program in the Higher Education in Indonesia, doi: 10.35940/ijrte.b1022.0982s919, Int. J. of Recent Technol. and Eng., 8(29), 1006-1010 (2019).

Sharafizad, J., Informal Learning of Women Small Business Owners, doi. 10.1108/et-01-2017-0006, Educ.+Training, 60(1), 82-103 (2017).

Ting, S.X., Feng L., y Qin W. The Effect of Entrepreneur Mentoring and its Determinants in the Chinese Context, doi: 10.1108/md-07-2016-0477, Manag. Decision, 55(7), 1410-1425 (2017).

Tinoco-Giraldo, H., Torrecilla, E.M. y García-Peñalvo, F.J. E-mentoring in Higher Education: a Structured Literature Review and Implications for Future Research, doi:10.3390/su12114344, Sustainability, 12(11),1-24. (2020). 
